\title{
Clustering with Multi-Layer Graphs: A Spectral Perspective
}

\author{
Xiaowen Dong, Pascal Frossard, Pierre Vandergheynst and Nikolai Nefedov
}

\begin{abstract}
Observational data usually comes with a multimodal nature, which means that it can be naturally represented by a multi-layer graph whose layers share the same set of vertices (objects) with different edges (pairwise relationships). In this paper, we address the problem of combining different layers of the multi-layer graph for an improved clustering of the vertices compared to using layers independently. We propose two novel methods, which are based on a joint matrix factorization and a graph regularization framework respectively, to efficiently combine the spectrum of the multiple graph layers, namely the eigenvectors of the graph Laplacian matrices. In each case, the resulting combination, which we call a "joint spectrum" of multiple layers, is used for clustering the vertices. We evaluate our approaches by experiments with several real world social network datasets. Results demonstrate the superior or competitive performance of the proposed methods compared to state-of-the-art techniques and common baseline methods, such as co-regularization and summation of information from individual graphs.
\end{abstract}

Index Terms-Multi-layer graphs, spectrum of the graph, matrix factorization, graph-based regularization, clustering.

\section{INTRODUCTION}

C LUSTERING on graphs is a problem that has been studied extensively for years. In this task we are usually given a set of objects, as well as an adjacency matrix capturing the pairwise relationships between these objects. This adjacency matrix is either represented by an unweighted graph, where the weight of the edges is always equal to one, or a weighted graph, where the weight can take any real positive values. The goal is to find an assignment of the objects into several subsets, such that the ones in the same subset are similar in some sense. Due to the wide range of applications for this problem, numerous approaches have been proposed in the literature, and we point the readers to the work of Schaeffer [1] for an extensive survey on this topic.

In contrast to the traditional problem, recent applications such as mobile and online social network analyses bring interesting new challenges. In these scenarios, it is common that

Copyright (c) 2012 IEEE. Personal use of this material is permitted. However, permission to use this material for any other purposes must be obtained from the IEEE by sending a request to pubs-permissions @ieee.org.

X. Dong is with Signal Processing Laboratories (LTS4 / LTS2), Institute of Electrical Engineering, Ecole Polytechnique Fédérale de Lausanne (EPFL), CH-1015 Lausanne, Switzerland (e-mail: xiaowen.dong@epfl.ch).

P. Frossard is with Signal Processing Laboratory (LTS4), Institute of Electrical Engineering, Ecole Polytechnique Fédérale de Lausanne (EPFL), CH-1015 Lausanne, Switzerland (e-mail: pascal.frossard@epfl.ch).

P. Vandergheynst is with Signal Processing Laboratory (LTS2), Institute of Electrical Engineering, Ecole Polytechnique Fédérale de Lausanne (EPFL), CH-1015 Lausanne, Switzerland (e-mail: pierre.vandergheynst@epfl.ch).

N. Nefedov is with Nokia Research Center (NRC), Lausanne, Switzerland (e-mail: nikolai.nefedov@nokia.com). observational data contains multiple modalities of information reflecting different aspects of human interactions. This can be conveniently represented by a multi-layer graph whose layers share the same set of vertices representing users, but have different sets of edges for different modalities. Fig. 1 [2] illustrates the mobile phone data collected in the MIT Reality Mining Project [3] as such a multi-layer graph. Specifically, the multiple layers represent relationships between mobile phone users in three different aspects: (i) Saturday night proximity, (ii) physical movement similarity, and (iii) interaction with phone communication. Intuitively, each layer should contribute to a meaningful clustering result from its own angle; however, one can expect that a proper combination of the three layers will possibly lead to improved clustering results by efficient merging and completion of data in each layer.

In this paper, we seek for such a good combination and propose two novel clustering methods by studying the spectrum of the graph. In particular, we propose two efficient ways to combine the spectrum of the multiple graph layers, which results in a "joint spectrum" that is eventually used for spectral clustering [4]. In more details, we first propose to generalize the eigen-decomposition process applied on a single Laplacian matrix to the case of multiple graph Laplacian matrices. We design a joint matrix factorization framework in which each graph Laplacian is approximated by a set of joint eigenvectors shared by all the graph layers, as well as its specific eigenvalues from the eigen-decomposition. These joint eigenvectors can then be used to form a joint low dimensional embedding of the vertices in the graph, based on which we perform clustering. In the second approach, we propose a graph regularization method that combines the spectrum of two graph layers. Specifically, we treat the eigenvectors of the Laplacian matrix from one graph as functions on the other graph. By enforcing the "smoothness" of such functions on the graph through a novel regularization framework, we are able to capture the characteristics of both graphs and achieve improved clustering results compared to using any graph alone. We finally propose an information-theoretic approach to generalize this second method to multiple graph layers.

We evaluate the performance of the proposed clustering methods on several real world social network datasets, and compare them with state-of-the-art techniques as well as several baseline methods used for graph-based clustering, such as summation of information from individual graphs. The results show that, in terms of three evaluation measures, our algorithms outperform the baseline methods, and are very competitive with the state-of-the-art technique introduced in Kumar et al. [5]. Furthermore, it is important to note that the 
Saturday night proximity

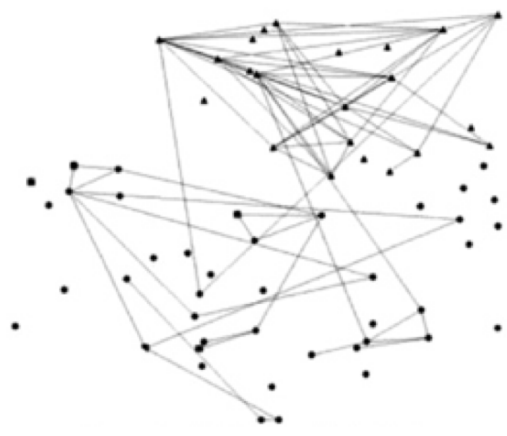

Cell tower transition

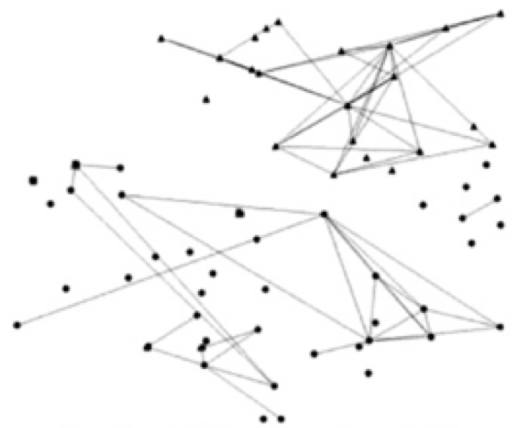

Phone communication

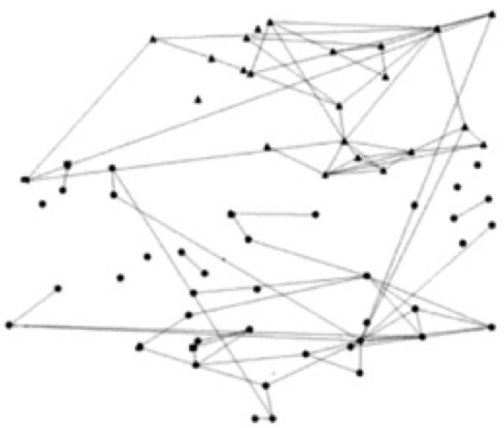

Fig. 1. A multi-layer graph in a mobile social network [2]. On the left, two mobile users are connected with an edge if they were proximate to each other during one Saturday night. In the middle, two are linked to each other if they made the same cell tower transitions at the same time. On the right, we assign an edge between each pair of users who interacted with phone communication.

Cell tower proximity

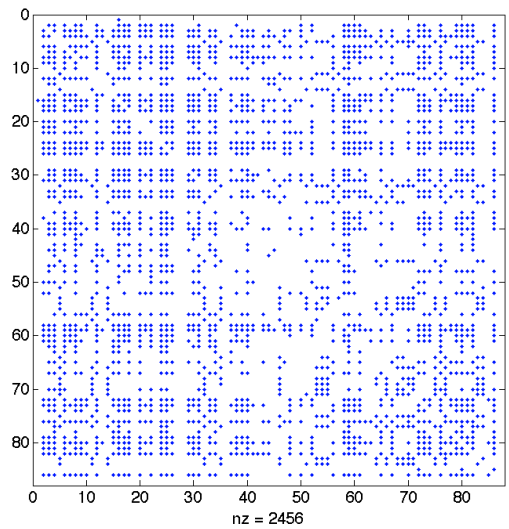

Bluetooth proximity

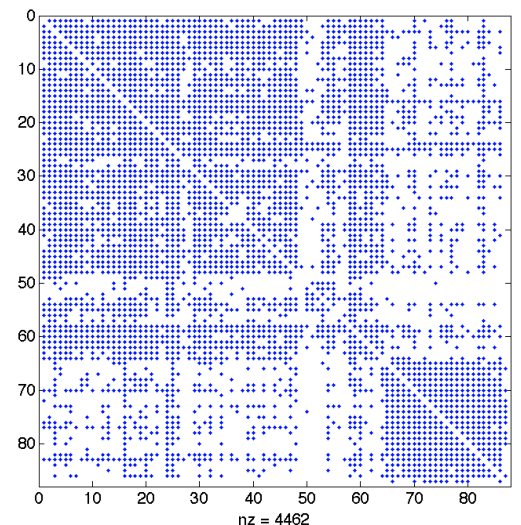

Phone calls

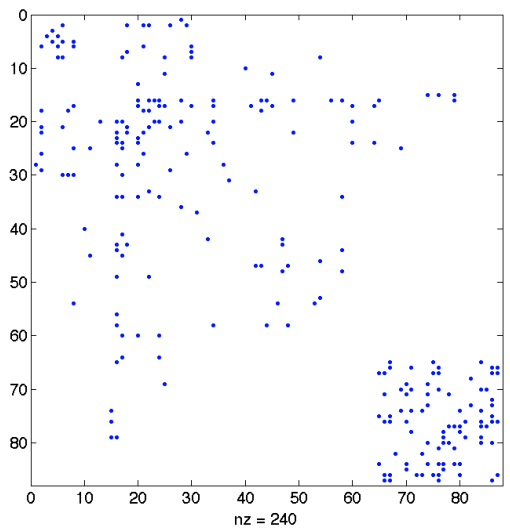

Fig. 2. Spy plots of three adjacency matrices from the MIT dataset. The redundant information contained in the layers for cell tower and bluetooth proximities can compensate the sparse information from the layer for phone call relationship for improved clustering results.

contribution of this paper is not limited to a better clustering with multiple graph layers. More generally, the concept of "joint spectrum" is helpful in the analysis of multimodal data that can be conveniently modeled as a multi-layer graph. As an example, it can lead to the generalization of the classical spectral analysis frameworks to multi-dimensional cases.

The rest of the paper is organized as follows. In Section II, we formally introduce the problem of clustering with multilayer graphs and motivate it from a practical example. In Section III, we review briefly the spectral clustering algorithm, which is one of the building blocks of the methodologies proposed in this paper. Next, we describe in details our novel multi-layer clustering algorithms in Section IV and Section V. We then provide experiments in Section VI, where we describe the datasets and present results and comparisons with the existing methods. Finally, we list the related work in Section VII and conclude the paper in Section VIII.

\section{Clunstering With MUlti-LAyer GRAPHS}

Consider a multi-layer graph $\mathcal{G}^{1}$ that contains $M$ individual graph layers $\mathcal{G}^{(i)}, i=1, \ldots, M$, where each layer $\mathcal{G}^{(i)}=\left\{V, E^{(i)}, \omega^{(i)}\right\}$ is a weighted and undirected graph consisting of a common vertex set $V$ and a specific edge set $E^{(i)}$ with associated weights $\omega^{(i)}$. Assuming that each layer reveals certain aspect of the intrinsic relationships between the vertices, one can expect that a proper combination of information contained in the multiple graph layers possibly leads to an improved unified clustering of the vertices in $V$. This can be further demonstrated by the following example.

Let us consider a three-layer graph built from the MIT Reality Mining Dataset [6], where the common vertex set represents 87 participants of the Reality Mining Project and edges represent relationships between these mobile phone users in three different aspects, namely, cell tower proximity, bluetooth proximity and phone call relationship. From these graph layers we form three adjacency matrices and depict them in the spy plots in Fig. 2, where each non-zero entry in the matrices corresponds to a point in the plots ${ }^{2}$. Intuitively,

\footnotetext{
${ }^{1}$ Throughout the paper, the notation $\mathcal{G}$ without upper index still represents a single graph unless stated differently.

${ }^{2}$ In these plots, the users are ordered according to six ground truth clusters. However, one may find that it is not easy to distinguish the clusters from the observations, which in fact demonstrates the difficulty of this clustering task. Detailed discussions are in Section VI
} 

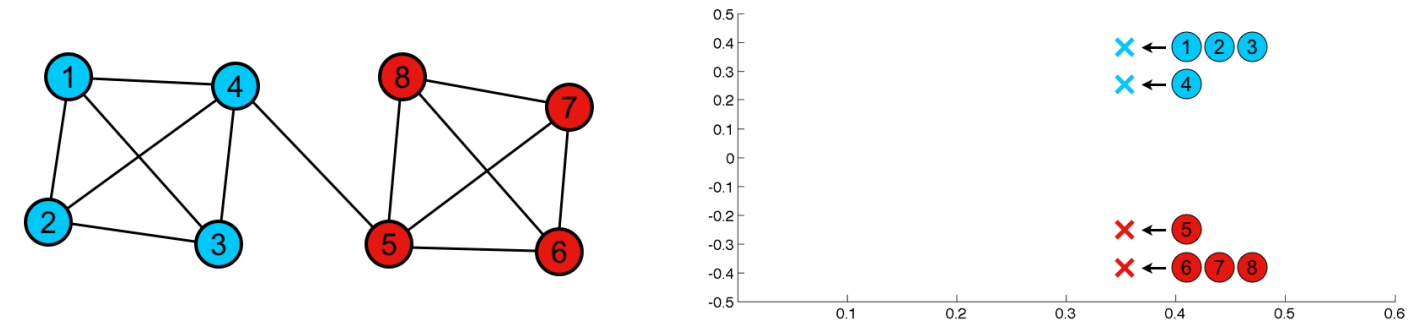

Fig. 3. Toy example to illustrate the spectral embedding. On the left is a simple unweighted graph with eight vertices, which we want to partition into two clusters. On the right is the embedding of the original vertices into a 2-dimensional space using the spectrum of the graph, where the coordinates on the horizontal and vertical axes are determined by the first and second eigenvectors of $L_{\mathrm{rw}}$. In this case, vertices 1 , 2 and 3 are embedded into the same point, and so are vertices 6,7 and 8 . It is clear to see that such an embedding helps reveal the intrinsic relationships between the vertices, and $k$-means can easily find the two clusters.

compared to the first two layers, entries in the phone call matrix are stronger indicators of friendship between users, hence the corresponding blue points in the third plot are more reliable. However, the sparse nature of this matrix makes it insufficient for achieving a good global clustering result for all the mobile users. In fact, this graph layer consists of many disconnected components, which makes it very difficult to assign cluster memberships to isolated vertices in the graph. In this case, the first two layers are more informative for achieving the clustering goal: even though single entries in those two matrices are less indicative, they provide richer structural information. This means that, by properly combining layers of different characteristics, we could expect an improved unified clustering result.

In this paper, we address the following problem. Given a multi-layer graph $\mathcal{G}$ with $M$ individual layers $\mathcal{G}^{(i)}, i=$ $1, \ldots, M$, we want to compute a joint spectrum that properly combines the information provided in different layers. In addition, the joint spectrum shall lead to an effective grouping of the vertices in $V$ with spectral clustering [4].

We propose two novel methods for the construction of a joint spectrum in the multi-layer graph.

\section{SPECTRAL CLUSTERING}

The idea of working with the spectrum of the graph is inspired by the popular spectral clustering algorithm [4]. In this section, we give a very brief review of this algorithm applied on a single graph, which is the main building block of our novel clustering algorithms. Readers familiar with spectral clustering could skip this section.

Spectral clustering has become increasingly popular due to its simple implementation and promising performance in many graph-based clustering problems. It can be described as follows. Consider a weighted and undirected graph $\mathcal{G}$. The spectrum of $\mathcal{G}$ is represented by the eigenvalues and eigenvectors of the graph Laplacian matrix $L=D-W$ where $W$ is the adjacency matrix and $D$ is the degree matrix containing degrees of vertices along diagonal. Notice that $L$ is also called the unnormalized or combinatorial graph Laplacian matrix. There are two normalized versions of the graph Laplacian defined as follows:

$$
\begin{aligned}
L_{\mathrm{sym}} & =D^{-\frac{1}{2}}(D-W) D^{-\frac{1}{2}}, \\
L_{\mathrm{rw}} & =D^{-1}(D-W),
\end{aligned}
$$

where $L_{\text {sym }}$ keeps the property of symmetry and $L_{\text {rw }}$ has close connection to the random walk process on graphs [7]. Different choices of the graph Laplacian correspond to different versions of the spectral clustering algorithm and detailed discussion on these choices is given in von Luxburg [7]. In this paper, we adopt the normalized spectral clustering algorithm that has been first described in Shi et al. [4]. It essentially corresponds to dealing with the eigenvalues and eigenvectors of the graph Laplacian $L_{\mathrm{rw}}$. In practice, the algorithm computes the spectrum of $\mathcal{G}$, and embeds the original vertices in $\mathcal{G}$ to a low dimensional spectral domain formed by the graph spectrum. Due to the properties of the graph Laplacian matrix, this transformation enhances the intrinsic relationships between the original vertices. Consequently, clusters can be eventually detected in the new low dimensional space by many common clustering algorithms, such as the $k$-means algorithm [8]. An example of such an embedding is illustrated in the toy example shown in Fig. 3. An overview of the algorithm is given in Algorithm 1.

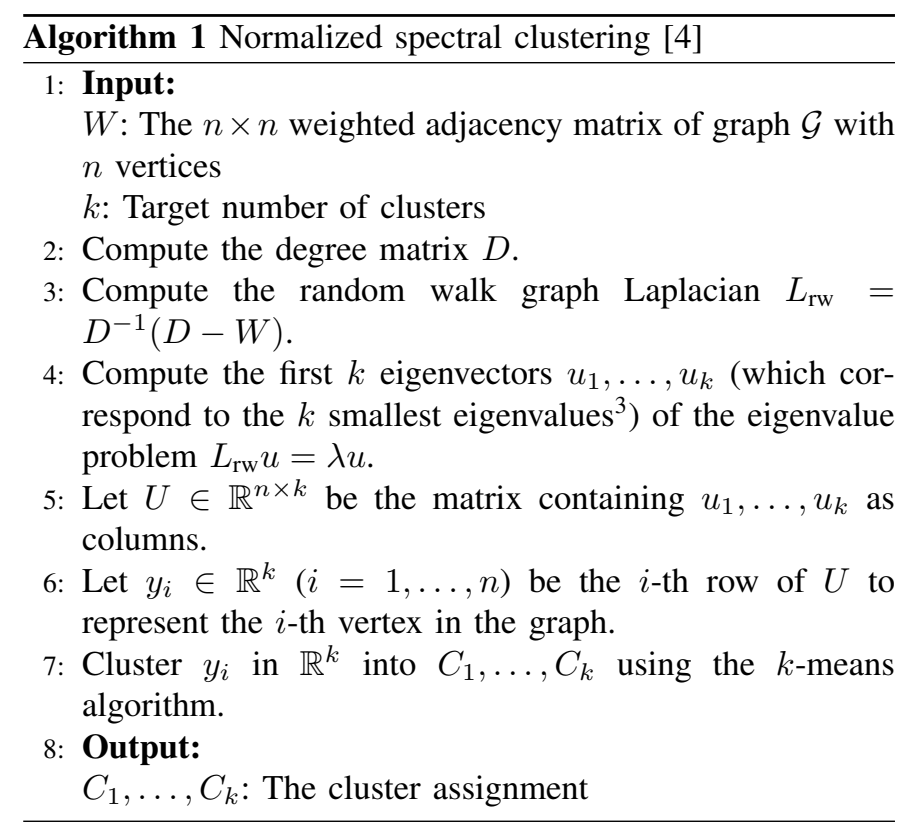

\footnotetext{
${ }^{3}$ Throughout the paper, eigenvalues and eigenvectors are always sorted in an ascending order, that is, $u_{1}$ is the eigenvector that corresponds to the smallest eigenvalue $\lambda_{1}$ and $u_{n}$ corresponds to the largest eigenvalue $\lambda_{n}$.
} 
As we can see in Algorithm 1, the spectral embedding matrix $U$ consisting of the first $k$ eigenvectors of the graph Laplacian represents the key idea of spectral clustering. It gives a new representation $y_{i}$ for each vertex in this low dimensional space, which makes the clustering task trivial with the $k$-means algorithm. Moreover, as theoretical guarantees, von Luxburg [7] shows that the effectiveness of this approach can be explained from the viewpoint of several mathematical problems, such as the normalized graph-cut problem [4], the random walk process on graphs [9] and problems in perturbation theory [10], [11]. In the following two sections, we will generalize this idea to the case of multi-layer graphs, where we aim at finding a joint spectrum to form the spectral embedding matrix that represents information from all the graph layers.

\section{Clustering With Generalized EIGEN-DECOMPOSITION}

The first method that we propose for clustering with multilayer graphs is built on the construction of an average spectral embedding matrix, based on which spectral clustering is eventually performed. We compute the average spectral embedding matrix with a generalized eigen-decomposition process. As we know, in order to compute the spectrum of a graph $\mathcal{G}$ with $n$ vertices, namely the eigenvalues and eigenvectors of its Laplacian matrix $L_{\mathrm{rw}}$, one can compute an eigen-decomposition of the matrix $L_{\mathrm{rw}}$ as:

$$
L_{\mathrm{rw}}=P \Lambda P^{-1},
$$

where $P$ is a $n \times n$ matrix containing eigenvectors of $L_{\mathrm{rw}}$ as columns, and $\Lambda$ is a $n \times n$ diagonal matrix containing the corresponding eigenvalues as the diagonal entries. In case of a multi-layer graph $\mathcal{G}$ with $n$ vertices, we have $M$ Laplacian matrices $L_{\mathrm{rw}}^{(i)}, i=1, \ldots, M$, one for each graph layer $\mathcal{G}^{(i)}$. As a natural extension, we propose to approximate each graph Laplacian $L_{\mathrm{rw}}^{(i)}$ by a set of joint eigenvectors shared by all the graph layers as well as its specific eigenvalue matrix:

$$
L_{\mathrm{rw}}^{(i)} \approx P \Lambda^{(i)} P^{-1} \text { for } i=1, \ldots, M,
$$

where $P$ is a $n \times n$ matrix containing the set of joint eigenvectors as columns, and $\Lambda^{(i)}$ is the $n \times n$ eigenvalue matrix of $L_{\mathrm{rw}}^{(i)}$. We now have to compute $P$, that is the set of eigenvectors that provides a good decomposition of the Laplacian matrix of all layers in the multi-layer graph. To do this, we propose to minimize the following objective function $S$, written as:

$$
\begin{aligned}
\arg \min _{P, Q \in \mathbb{R}^{n \times n}} S= & \frac{1}{2} \sum_{i=1}^{M}\left\|L_{\mathrm{rw}}^{(i)}-P \Lambda^{(i)} Q\right\|_{F}^{2} \\
& +\frac{\alpha}{2}\left(\|P\|_{F}^{2}+\|Q\|_{F}^{2}\right)+\frac{\beta}{2}\left\|P Q-I_{n}\right\|_{F}^{2},
\end{aligned}
$$

where $P$ represents the joint eigenvector matrix, $Q$ is enforced to be the inverse matrix of $P$ so that it plays the role of $P^{-1}$ in Eq. (4), and $\Lambda^{(i)}$ captures the characteristic of the $i$-th graph layer $\mathcal{G}^{(i)}$. In addition, $I_{n}$ represents the identity matrix of dimension $n$ and $\|\cdot\|_{F}$ denotes the Frobenius norm. Hence, the first term of the objective function $S$ is a data fidelity term to measure the overall approximation error when all layers are decomposed over $P$; the second term, the sum of the squared Frobenius norms of $P$ and $Q$, is added to improve numerical stability of the solution; and the third term is a constraint to enforce $Q$ to be the inverse of $P$. Notice that the purpose of introducing the additional variable $Q$ is purely for the computational convenience of the optimization process. Finally, the regularization parameters $\alpha$ and $\beta$ balance the trade-offs between the three terms in the objective function.

Now we have to solve the problem in Eq. (5) to get $P$. Since the objective $S$ is not jointly convex in $P$ and $Q$, it is difficult to find the global minimum solution to Eq. (5). Therefore, we adopt an alternating scheme to find a local minimum of the objective function. In the outer loop, we first fix $Q$ and optimize $P$, and then optimize $Q$ while fixing $P$. As a consequence, it is important to give a good initialization to our algorithm. In practice, we suggest to compute the eigen-decomposition of $L_{\mathrm{rw}}^{(1)}$ from the most informative graph layer $\mathcal{G}^{(1)}$, and initialize $P$ as the matrix containing its eigenvectors as columns. $Q$ is initialized as the inverse of $P$. The optimization process is then repeated until the stopping condition is satisfied. In the inner loop, we solve each variable while the other is being fixed. Notice that the objective function $S$ is differentiable with respect to variables $P$ and $Q$ :

$$
\begin{gathered}
\frac{\partial S}{\partial P}=-\sum_{i=1}^{M}\left(L_{\mathrm{rw}}^{(i)}-P \Lambda^{(i)} Q\right) Q^{T} \Lambda^{(i)}+\alpha P+\beta\left(P Q-I_{n}\right) Q^{T} \\
\frac{\partial S}{\partial Q}=-\sum_{i=1}^{M}\left(L_{\mathrm{rw}}^{(i)}-P \Lambda^{(i)} Q\right) P \Lambda^{(i)}+\alpha Q+\beta\left(P Q-I_{n}\right) P,
\end{gathered}
$$

where $(\cdot)^{T}$ denotes the matrix transpose operator. Therefore, we use an efficient quasi-Newton method (Limited-Memory BFGS [12]) to solve each variable.

We have now computed $P$, which is the set of joint eigenvectors, namely a joint spectrum shared by the multiple graph layers. The average spectral embedding matrix is then formed by the first $k$ joint eigenvectors, that is, the first $k$ columns of $P$. We then follow the steps 6 and 7 in Algorithm 1 to eventually perform the clustering. The corresponding algorithm is given in Algorithm 2.

Notice that the algorithm proposed in this section is in a sense similar to the approach in Tang et al. [13], which introduces a matrix factorization framework to find a low rank matrix that is shared by all the graph layers. However, the matrices they try to approximate in their work are not the graph Laplacian matrices, but the adjacency matrices of all the layers. Moreover, note that the generalized eigen-decomposition process above is essentially based on averaging the information from the multiple graph layers. It tends to treat each layer equally and to build a solution that might smooth out the specificities of each layer. In the next section, we propose a second method based on a regularization process between different layers, which is able to preserve the particularities of each individual layer. 


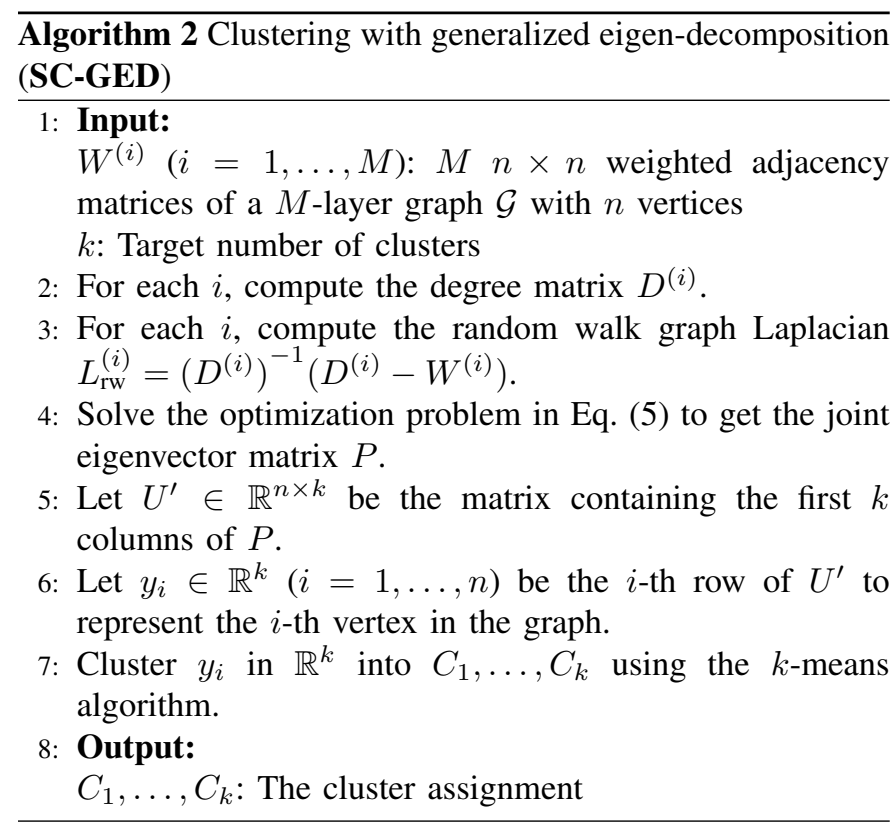

\section{Clustering With SPECTRAL REgularization}

In this section, we propose the second novel method for clustering with multi-layer graphs, where we treat all layers based on their respective importance. As a consequence, this method helps preserve specificities of each layer in the clustering process.

\section{A. Intuition}

We first examine the behavior of eigenvectors of the graph Laplacian matrix in more details. Consider a weighted and connected graph $\mathcal{G}$ with vertex set $V=\left\{v_{i}, i=1, \ldots, n\right\}$. From spectral graph theory [14], we know that the eigenvectors $u_{1}, \ldots, u_{n}$ of the graph Laplacian matrix $L$ have the following properties:

1) The first eigenvalue $\lambda_{1}$ is 0 and the corresponding eigenvector $u_{1}$ is the constant one vector $\mathbf{1}$;

2) For $i=2, \ldots, n, u_{i}$ satisfies: $u_{i} \perp \mathbf{1}$ and $\left\|u_{i}\right\|=1$ (after normalization).

Now consider the problem of mapping the graph $\mathcal{G}$ on a 1dimensional line such that connected vertices stay as close as possible on the line, with the condition that the mapping vector satisfies the second property above. In other words, we want to find a 1-dimensional mapping (or a scalar function) $f: V \rightarrow R$ that minimizes the following term:

$$
\begin{aligned}
\arg \min _{f \in \mathbb{R}^{n}}\left\{\sum_{i, j}^{n} w_{i, j}\left(f\left(v_{i}\right)-f\left(v_{j}\right)\right)^{2}\right\}, \\
\text { s.t. } \quad f \perp \mathbf{1},\|f\|=1,
\end{aligned}
$$

where $f\left(v_{i}\right)$ and $f\left(v_{j}\right)$ represent the mappings of vertices $v_{i}$ and $v_{j}$ respectively, and $w_{i, j}$ is the weight of the edge between the two vertices. The constraints on the norm of $f$ and the orthogonality to the constant one vector $\mathbf{1}$ are introduced to make the solution nontrivial and unique, and can be explained from a graph-cut point of view [7]. Moreover, since eigenvectors of the Laplacian matrix can be viewed as scalar functions defined on the vertices of the graph, these conditions suggest that they can be considered as candidate solutions to the problem in Eq. (8). In fact, we can rewrite Eq. (8) in terms of the graph Laplacian matrix $L$ so that an equivalent problem is:

$$
\arg \min _{f \in \mathbb{R}^{n}} f^{T} L f, \quad \text { s.t. } \quad f \perp \mathbf{1},\|f\|=1 .
$$

It can be shown by the Rayleigh-Ritz theorem [7] that the solution to the problem in Eq. (9) is $u_{2}$, the eigenvector that corresponds to the second smallest eigenvalue of $L$, which is usually called the Fiedler vector of the graph.

Illustrative examples of such a mapping can be found in Horaud et al. [15]-[17], which shows that the mapping of $u_{2}$ indeed keeps the strongly connected vertices as close as possible on the line. More importantly, it is shown in Zhou et al. [18] that the quadratic objective in Eq. (9) can be viewed as a smoothness measure of the scalar function $f$ defined on the vertices of the graph $\mathcal{G}$, that is, $f$ has similar values on the vertices that are strongly connected in the graph. Therefore, the fact that it minimizes this objective implies that the Fiedler vector $u_{2}$ is a smooth function on the graph. In fact, since we have

$$
u_{i}^{T} L u_{i}=\lambda_{i}, \quad \text { for } \quad i=2, \ldots, n,
$$

all the first $k$ eigenvectors tend to be smooth on the graph $\mathcal{G}$ provided that the first $k$ eigenvalues are sufficiently small. Since these first $k$ eigenvectors are used to form the low dimensional embedding $U$ in the spectral clustering algorithm, such smoothness property implies that a special set of smooth functions on the graph, such as eigenvectors of the graph Laplacian matrix, can well represent the graph connectivity and hence help in the clustering process.

This inspires us to combine information from multiple graph layers, with the help of a set of joint eigenvectors that are smooth on all the layers, hence capture all their characteristics. However, instead of treating all the layers equally, we try to highlight the specificities of different layers. Therefore, we propose the following methodology. Consider two graph layers $\mathcal{G}^{(1)}$ and $\mathcal{G}^{(2)}$ in a 2-layer graph. From the smoothness analysis above, we observe that the eigenvectors of the Laplacian matrix from $\mathcal{G}^{(1)}$ are smooth functions on $\mathcal{G}^{(1)}$; in the meantime, since they can also be viewed as scalar functions on $\mathcal{G}^{(2)}$, we try to enforce their smoothness on $\mathcal{G}^{(2)}$ as well. This leads to a set of eigenvectors that are smooth on both layers, namely a jointly smooth spectrum shared by $\mathcal{G}^{(1)}$ and $\mathcal{G}^{(2)}$; this spectrum captures the characteristics of both layers in this 2-layer graph.

\section{B. Jointly smooth spectrum computation}

We propose a spectral regularization process to compute a jointly smooth spectrum of two graph layers $\mathcal{G}^{(1)}$ and $\mathcal{G}^{(2)}$ by solving the following optimization problem:

$$
\arg \min _{f_{i} \in \mathbb{R}^{n}}\left\{\frac{1}{2}\left\|f_{i}-u_{i}\right\|_{2}^{2}+\lambda \cdot \Phi_{f_{i}}\right\} \quad \text { for } \quad i=2, \ldots, n,
$$

where $f_{i}: V \rightarrow R$ is a scalar function on the graph, $u_{i}$ is the $i$-th eigenvector from $\mathcal{G}^{(1)}$, and $\Phi_{f_{i}}=f_{i}^{T} L_{\mathrm{sym}}^{(2)} f_{i}$ is a quadratic term ${ }^{4}$ from $\mathcal{G}^{(2)}$ which measures the smoothness of 
$f_{i}$ on $\mathcal{G}^{(2)}$. In the problem in Eq. (11), we seek for a scalar function $f_{i}$ such that it is not only close to the eigenvector $u_{i}$ that comes from $\mathcal{G}^{(1)}$, but also sufficiently smooth on $\mathcal{G}^{(2)}$ in terms of the quadratic smoothness measure. This promotes the smoothness property of our solution $f_{i}$ on both of the layers, so that $f_{i}$ can be considered as a joint eigenvector of $\mathcal{G}^{(1)}$ and $\mathcal{G}^{(2)}$. The regularization parameter $\lambda$ is used to balance the trade-off between the data fidelity term and the regularization term in the objective function.

It is shown that the problem in Eq. (11) has a closed form solution [18]:

$$
f_{i}^{*}=\mu\left(L_{\mathrm{sym}}^{(2)}+\mu I_{n}\right)^{-1} u_{i}
$$

where $\mu=\frac{1}{\lambda}$. Furthermore, notice that for each $u_{i}$ there is an associated optimization problem (except for $i=1$ since the first eigenvector is a constant vector), hence by solving all these problems we get a set of joint eigenvectors $f_{i}$, $i=2, \ldots, n$. Therefore, they together can be viewed as a jointly smooth spectrum of $\mathcal{G}^{(1)}$ and $\mathcal{G}^{(2)}$. The first $k$ joint eigenvectors can then be used to form a spectral embedding matrix, based on which we perform clustering. The overall clustering algorithm is summarized in Algorithm 3.

It is worth noting that $\mathcal{G}^{(1)}$ and $\mathcal{G}^{(2)}$ play different roles in our framework. Specifically, $\mathcal{G}^{(1)}$ is used for the eigendecomposition process to get the eigenvectors, and $\mathcal{G}^{(2)}$ is used as the graph structure for the regularization process. It is natural to choose the more informative layer as $\mathcal{G}^{(1)}$. Moreover, we can generalize the above framework to multi-layer graphs with more than two layers. Specifically, we propose to start with the most informative graph layer $\mathcal{G}^{(1)}$, and search for the next layer $\mathcal{G}^{(2)}$ that maximizes the mutual information between $\mathcal{G}^{(1)}$ and $\mathcal{G}^{(2)}$. More clearly, the mutual information between two graph layers is introduced by interpreting clustering from each individual layer as a discrete distribution of the cluster memberships of the vertices. Therefore, it can be calculated by measuring the mutual information between two distributions using Eq. (20). Next, after having the combination of the first two layers, we can repeat the process by maximizing the mutual information between the current combination and the next selected layer, until we include all the layers in the end. This provides a greedy approach to compute a jointly smooth spectrum of all layers in the multi-layer graph.

\section{Discussion}

In addition to the intuition provided above, we further explain in this subsection why the spectral regularization process is considered as a good way of combining the spectrum of two graph layers.

We first interpret the combination of multiple layers from the viewpoint of label propagation [19]-[22], which is proven to be an effective approach for graph-based semi-supervised learning. In label propagation, one usually has a similarity graph whose vertices represent objects and edges reflect the pairwise relationships between them. We let the initial labels

\footnotetext{
${ }^{4}$ Since the smoothness analysis in Section V-A can be easily generalized from $L$ to $L_{\text {sym }}$, here we follow Zhou et al. [18] to use $L_{\text {sym }}$ instead of $L$ for a better implementation of the algorithm.
}

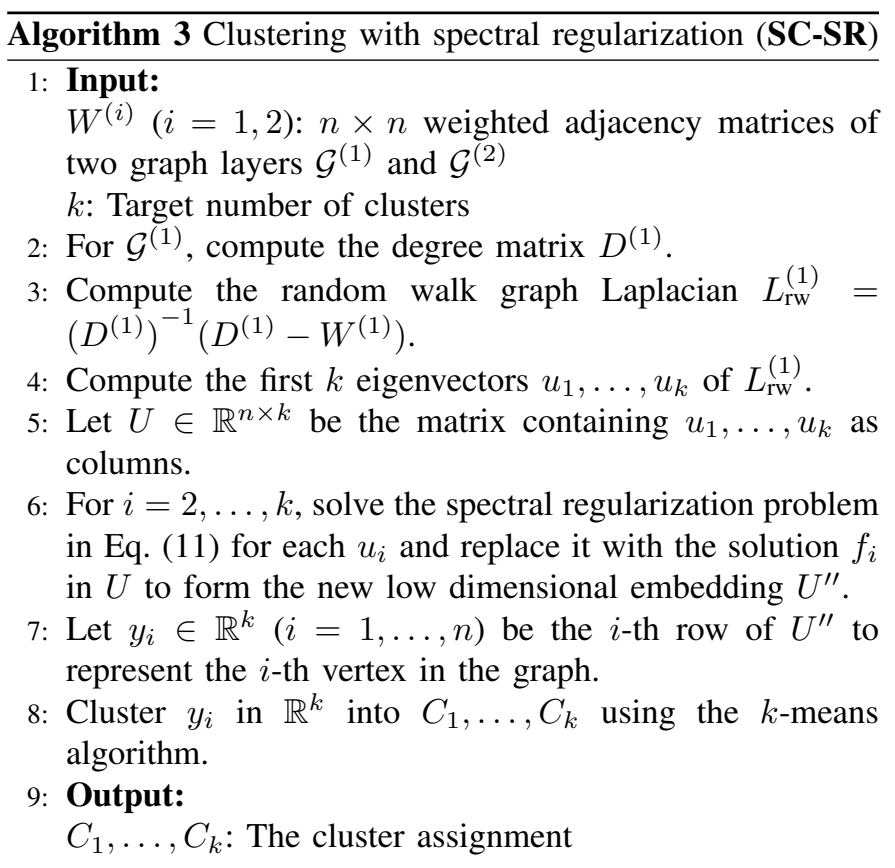

of the vertices propagate towards their neighboring vertices to make inference, based on the strength of relationships between them and their neighbors. This is exactly what the spectral regularization process in Eq. (11) does. More clearly, the optimization problem in Eq. (11) can be solved through an iterative process, where in each iteration we have for every vertex $v \in V$ :

$$
\left(f_{i}(v)\right)^{[t+1]} \leftarrow \alpha\left(\left(I-L_{\mathrm{sym}}^{(2)}\right) f_{i}^{[t]}\right)(v)+(1-\alpha) u_{i}(v),
$$

where $u_{i}$ contains the initial values at the vertices and $f_{i}^{[t]}$ represents $f_{i}$ at iteration $t$ [18]. The parameter $\alpha$ is defined as $\alpha=\frac{\lambda}{1+\lambda}$ while $\lambda$ is the regularization parameter in Eq. (11). In other words, the value at each vertex is updated by a convex combination of the initial value $u_{i}(v)$ and the values at its neighboring vertices at the current iteration, where the parameter $\alpha$ balances the trade-off between the two portions. Notice that the initial values $\left\{u_{i}\right\}_{i=1}^{k}$ from $\mathcal{G}^{(1)}$ form the continuous-valued solver of a relaxed discrete graph-cut problem [7]. Therefore, $\left\{u_{i}\right\}_{i=1}^{k}$ can be viewed as labels indicating the cluster memberships derived from $\mathcal{G}^{(1)}$. Consequently, the spectral regularization process in Eq. (11) can be interpreted as a label propagation process, where the cluster labels derived from $\mathcal{G}^{(1)}$ are linearly propagated on $\mathcal{G}^{(2)}$. In this way, both of the graph structures have been taken into account hence making the resulting combination meaningful.

Another interpretation is based on disagreement minimization [5], [23], which has been proposed for the task of learning with multiple sources of data. The basic idea is to minimize the disagreement between information from the multiple sources so that we get a good combination of all the sources. For example, Kumar et al. [5] suggests a clustering algorithm that minimizes the disagreement between information from multiple graphs. Similarly, since we aim at finding a unified clustering result from multiple graph layers, it is natural to 
enforce the consistency between the clustering result and the information from all the graph layers, or in other words, to minimize the disagreement between them. Such a disagreement is again reflected in the objective function of the optimization problem in Eq. (11). More specifically, the data fidelity term explicitly measures the disagreement between the solution $f_{i}$ and the initial vector $u_{i}$ that comes from $\mathcal{G}^{(1)}$, while the regularization term implicitly represents the inconsistency of the information contained in $f_{i}$ with the structure of $\mathcal{G}^{(2)}$. Indeed, the regularization term $\Phi_{f}$ can be expressed in the following form:

$$
\Phi_{f}=\frac{1}{2} \sum_{i, j}^{n} w_{i, j}\left(\frac{f\left(v_{i}\right)}{\sqrt{d\left(v_{i}\right)}}-\frac{f\left(v_{j}\right)}{\sqrt{d\left(v_{j}\right)}}\right)^{2},
$$

where $d\left(v_{i}\right)$ and $d\left(v_{j}\right)$ are the degrees of $v_{i}$ and $v_{j}$, respectively. This means that $\Phi_{f_{i}}$ will only be small if the two end-point vertices of a large-weight edge in $\mathcal{G}^{(2)}$ have similar function values normalized by their degrees. Therefore, minimizing the objective function in Eq. (11) can be considered as minimizing the total disagreement between the solution $f_{i}$ and the information from multiple graph layers. Notice that in this formation the disagreement is modeled from two different viewpoints for the two individual layers, whose respective importance is controlled by the parameter $\lambda$.

\section{EXPERIMENTAL RESULTS}

In this section we present the experimental results. We first describe the datasets and different clustering algorithms used in the experiments, and then compare their performances in terms of three evaluation measures for clustering.

\section{A. Datasets}

We adopt three real world social network datasets to compare the clustering performances between our proposed methods and the existing approaches. Two of them are mobile phone datasets, and the third one is a bibliographic dataset. In this section, we give a brief description on each dataset and explain how we construct multiple graph layers in each case.

The first dataset is a subset of the MIT Reality Mining Dataset $^{5}$, which includes mobile phone data of 87 mobile users on the MIT campus. We select three types of information to build a multi-layer graph: physical locations, bluetooth scans and phone calls. More specifically, for physical locations and bluetooth scans, we measure how many times two users are under the service of the same cell tower, and how many times two have scanned the same bluetooth device, within a 30minute time window. Aggregating results from such windows throughout a 10-month period gives us two weighted adjacency matrices. In addition, a phone call matrix is generated by assigning weight of the edge between any two users as how many times one has established or received calls from the other. In this dataset, we take the ground truth of the clusters as the self-reported affiliations of the subjects. The clustering objective is to partition all the users into six groups with the 3-layer graph and compare them with the six ground truth clusters.

\footnotetext{
${ }^{5}$ Available online at "http://reality.media.mit.edu/download.php".
}

The second dataset in our experiments is the mobile phone dataset that is currently being collected by Nokia Research Center (NRC) Lausanne [24], which includes data of around 200 mobile users living or working in the area of Lausanne, Switzerland. We construct a multi-layer graph from the same information as that in the MIT dataset, with the only difference being that we measure the physical proximity between every pair of users directly using their GPS coordinates. In the Nokia dataset, we take the ground truth of the clusters as eight groups divided by their email affiliations. The goal is to find the ground truth clusters with the 3-layer graph constructed.

The third dataset we adopt is the Cora dataset ${ }^{6}$. Although the objects in this bibliographic dataset are research papers rather than people, it still reflects human interactions in research and publishing activities. In our experiments, we select 292 research papers that roughly come from three different fields: Natural Language Processing, Data Mining and Robotics. Each paper has been manually labeled with one of the categories and we consider this information as the ground truth of the clusters. To build the first two graph layers, we represent the title and abstract of each paper as vectors of nontrivial words, and take the cosine similarity between each pair of vectors as the corresponding entry in the adjacency matrices. In addition, we include a citation graph as the third layer that reflects the citation relationships of these papers. Finally, the goal is to cluster these papers based on the three graph layers we create.

It can be noted that the Cora dataset is considered quite easy to cluster while the MIT and Nokia datasets are much more difficult. The reason is that it is not straightforward to define the ground truth clusters for a group of human users, and observational data does not necessarily correspond well to the intended clusters. In these two datasets, both the academic and email affiliations are not fully reflected by the physical proximity and phone communication between the mobile users, which makes the tasks difficult. Moreover, as we can imagine, the Nokia dataset is expected to be even more difficult to cluster than the MIT dataset as email affiliations is less trustworthy. Nevertheless, we still choose the ground truth clusters in these ways as they are the best indicative information available in the datasets. After all, these two datasets are representative for analyses of activities of mobile phone users, and they can serve as challenging tasks in the experiments compared to the easier one from the Cora dataset.

\section{B. Clustering algorithms}

In this section, we explain briefly the clustering algorithms that are included in the performance comparisons, along with some implementation details. First of all, we describe some implementation details of the two proposed methods:

- SC-GED: Spectral clustering with generalized eigendecomposition described in Section IV. In SC-GED, there are two regularization parameters $\alpha$ and $\beta$ to balance the trade-off between the approximation error and the stability and conditions on the solution. In our experiments, we

\footnotetext{
${ }^{6}$ Available online at "http://www.cs.umass.edu/ mccallum/data.html" under category "Cora Research Paper Classification".
} 
set $\beta$ to be rather large, for example 100, to enforce the inverse relationship between $P$ and $Q$. We choose $\alpha$ to be 0.5 for the Nokia dataset and around 10 for the other two datasets.

- SC-SR: Spectral clustering with spectral regularization described in Section V. Since SC-SR is a recursive approach, we need to select two graph layers to fit in the regularization framework at each time. As discussed in Section V-B, we investigate the mutual information between different graph layers. As an example, in the MIT dataset, the "cell tower" and "bluetooth" layers have the highest mutual information between them. Therefore we choose to first combine these two layers. We select the "bluetooth" layer to act as $\mathcal{G}^{(1)}$ in the spectral regularization framework, as it is considered more informative than the "cell tower" layer. After the first combination, the third layer "phone call" is incorporated to get the final solution. In addition, at each combination step, there is a regularization parameter $\lambda$ in the optimization problem in Eq. (11) to control the relative importance of the two graph layers. Intuitively, the choice of this parameter at each step should loosely reflect the mutual information between the two layers being considered. We use this as a rule of thumb to set the parameters in the first and second combination steps, which are denoted by $\lambda_{1}$ and $\lambda_{2}$, respectively. As an example, we set $\lambda_{1}=2$ and $\lambda_{2}=1$ for the MIT dataset.

Next, we introduce five competing schemes as follows. The first three are common baseline methods for clustering with multiple graphs, and the last two are representative techniques in the literature:

- SC-SUM: Spectral clustering applied on the summation of the adjacency matrices:

$$
\sum_{i=1}^{M} W^{(i)}
$$

If the weights of edges are of different scales across the multiple layers, we use the summation of the normalized adjacency matrices:

$$
\sum_{i=1}^{M}\left(D^{(i)}\right)^{-\frac{1}{2}} W^{(i)}\left(D^{(i)}\right)^{-\frac{1}{2}} .
$$

- K-Kmeans: Kernel $k$-means applied on the summation of the spectral kernels of the adjacency matrices [13]:

$$
\sum_{i=1}^{M} K^{(i)} \quad \text { with } \quad K^{(i)}=\sum_{k=1}^{d} u_{k}^{(i)}\left(u_{k}^{(i)}\right)^{T},
$$

where $d \ll n$ (number of vertices) and $u_{k}^{(i)}$ represents the $k$-th eigenvector of the Laplacian $L_{\text {sym }}^{(i)}$ from $\mathcal{G}^{(i)}$.

- SC-AL: Spectral Clustering applied on the averaged random walk graph Laplacian matrix:

$$
\frac{1}{M} \sum_{i=1}^{M} L_{\mathrm{rw}}^{(i)}
$$

- Co-regularization (CoR): The co-regularization approach proposed in Kumar et al. [5] is the latest state-of-the-art technique aimed at combing information from multiple graphs. In this work, the authors have proposed to enforce the similarity between information from two different graphs where the similarity is measured by a linear kernel. In our experiments, we generalize their approach to multiple graphs and tune the hyperparameter $\lambda$ in their work to achieve the best clustering performance.

- Community detection via modularity maximization (CD): In addition to spectrum-based clustering algorithms, modularity maximization is a different approach proposed by Newman et al. [25]-[27] for community detection. We adopt the algorithm described in Nefedov [28], which applies modularity maximization [27] using fast greedy search algorithm [29]. It uses the summation of the normalized adjacency matrices to combine information from different graph layers.

\section{Evaluation measures and results}

To quantitatively evaluate the clustering performance, we compare the clusters $\Omega=\left\{\omega_{1}, \ldots, \omega_{k}\right\}$ we have computed with the intended ground truth classes $C=\left\{c_{1}, \ldots, c_{k}\right\}$. We adopt Purity, Normalized Mutual Information (NMI) and Rand Index (RI) [30] as three measures to evaluate the clustering performance from different angles. More specifically, Purity is defined as:

$$
\operatorname{Purity}(\Omega, C)=\frac{1}{n} \sum_{k} \max _{j}\left|\omega_{k} \cap c_{j}\right|,
$$

where $n$ is the total number of objects, and $\left|\omega_{k} \cap c_{j}\right|$ denotes the number of objects in the intersection of $\omega_{k}$ and $c_{j}$. Next, $N M I$ is defined as:

$$
N M I(\Omega, C)=\frac{I(\Omega ; C)}{[H(\Omega)+H(C)] / 2},
$$

where $I$ is the mutual information between clusters $\Omega$ and classes $C$, and $H(\Omega)$ and $H(C)$ represent the entropies of the clusters and classes, respectively. Finally, when interpreting clustering as a series of binary decisions on each pair of objects, $R I$ is defined as:

$$
R I(\Omega, C)=\frac{T P+T N}{T P+F P+F N+T N},
$$

where $T P, T N, F P, F N$ represent true positive, true negative, false positive and false negative decisions, respectively.

Fig. 4 shows the performance of different clustering algorithms applied on the three datasets we adopt. As we can see, clustering with the Cora dataset is indeed much easier than the other two datasets as the evaluation scores are much higher. Regarding the performance of the algorithms, it is clearly shown that proper combinations of multiple graph layers indeed lead to improved clustering results compared to using layers independently. In general, our proposed algorithm SC-SR achieves superior or competitive performances in all the evaluation measures, while SC-GED does not perform as well as SC-SR. Among the competitors, CoR presents impressive evaluation scores, while $\mathbf{C D}$ and the three baseline combination methods show intermediate results in general. As 


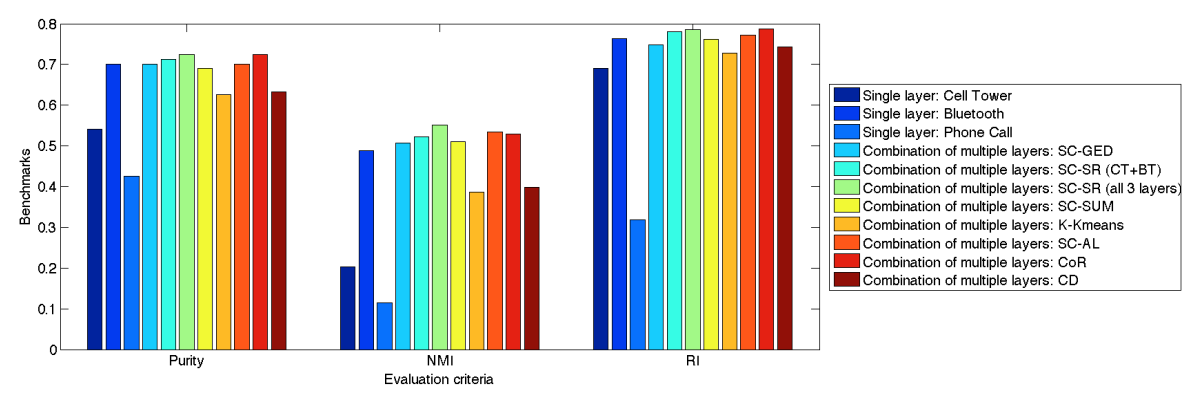

(a) Clustering performance on the MIT dataset

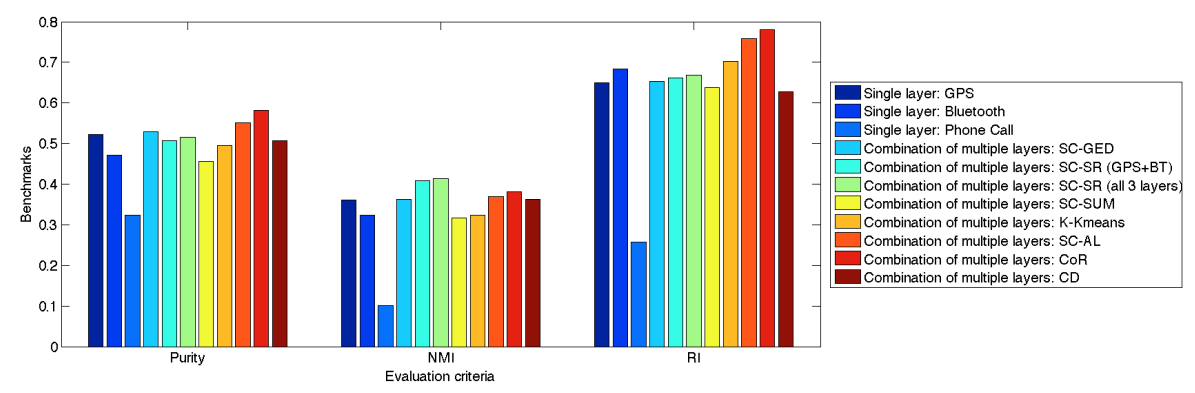

(b) Clustering performance on the Nokia dataset

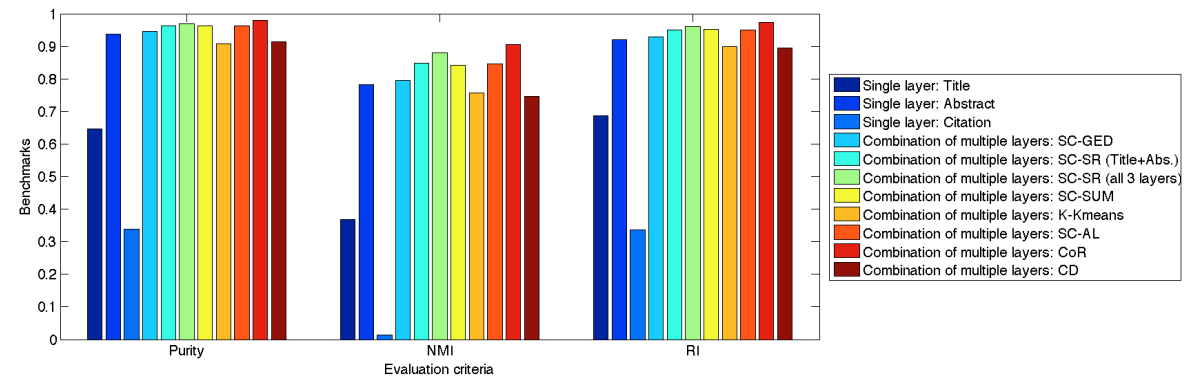

(c) Clustering performance on the Cora dataset

Fig. 4. Performance evaluation of different clustering algorithms.

we can imagine, this is mainly due to the simple averaging of the information from different graph layers.

In more details, we can see that the regularized combinations in SC-SR consistently lead to better clustering results as more layers are combined, particularly in terms of the NMI scores. This comes from the way we combine the multiple graph layers in order to maximize the mutual information. Compared to the state-of-the-art algorithm CoR, SC-SR maintains competitive results while the computational complexity is significantly reduced. Indeed, CoR needs to compute extremal eigenvectors of the (original and modified) Laplacian matrices for $M N$ times in total, where $M$ is the number of different graphs and $N$ is the number of iterations the algorithm needs to converge. In contrast, SC-SR only needs to implement the same process once, namely for the most informative layer. Therefore, with state-of-the-art eigensolvers [31], SC-SR has a complexity usually lower than $O\left(n^{3}\right)$, where $n$ is the number of vertices. Finally, notice that the NMI score shows difference from the other two measures in the Nokia dataset, since the ground truth clusters in this dataset are quite unbalanced.

Compared to SC-SR, the performance of SC-GED is somewhat disappointing, as it only provides limited improvements on the clustering results achieved by individual layers. This is mainly due to the nature of the algorithm: unlike SC-SR which is implemented recursively, it resorts to a joint matrix factorization framework to find the set of joint eigenvectors all at once. Therefore, it can be essentially considered as a way to average the information from the multiple graph layers, but without paying much attention to the specific characteristics in each layer. Moreover, the computational complexity of SCGED is higher than that of SC-SR, as we need to compute a complete eigen-decomposition and a matrix inversion (both with a complexity of $O\left(n^{3}\right)$ ) for the initialization, as well as for the evaluations of derivatives in Eq. (6) and Eq. (7) in the L-BFGS algorithm.

Finally, in addition to the evaluation scores, the confusion matrices for different clustering methods on the MIT dataset are shown in Table I as an illustrative example of the clustering 
TABLE I

CONFUSION MATRICES FOR SEVEN COMBINATION METHODS ON THE MIT DATASET.

\begin{tabular}{|c|c|c|c|c|c|}
\hline \multicolumn{6}{|c|}{ SC-GED } \\
\hline 4 & 7 & 0 & 1 & 3 & 0 \\
\hline 7 & 15 & 0 & 5 & 7 & 0 \\
\hline 0 & 1 & 5 & 0 & 0 & 0 \\
\hline 0 & 3 & 0 & 0 & 0 & 0 \\
\hline 0 & 6 & 0 & 0 & 0 & 0 \\
\hline 0 & 1 & 0 & 0 & 0 & 22 \\
\hline
\end{tabular}

\begin{tabular}{|c|c|c|c|c|c|}
\hline \multicolumn{6}{|c|}{ SC-SR (all 3 layers) } \\
\hline 6 & 3 & 0 & 1 & 5 & 0 \\
\hline 7 & 10 & 0 & 4 & 13 & 0 \\
\hline 0 & 0 & 6 & 0 & 0 & 0 \\
\hline 1 & 0 & 0 & $\mathbf{0}$ & 2 & 0 \\
\hline 1 & 0 & 0 & 0 & 5 & 0 \\
\hline 0 & 0 & 0 & 0 & 0 & 23 \\
\hline
\end{tabular}

SC-SUM

\begin{tabular}{|c|c|c|c|c|c|}
\hline $\mathbf{2}$ & 9 & 3 & 1 & 0 & 0 \\
\hline 8 & $\mathbf{2 0}$ & 3 & 1 & 2 & 0 \\
\hline 0 & 0 & $\mathbf{6}$ & 0 & 0 & 0 \\
\hline 0 & 2 & 1 & $\mathbf{0}$ & 0 & 0 \\
\hline 0 & 5 & 1 & 0 & $\mathbf{0}$ & 0 \\
\hline 0 & 0 & 0 & 0 & 0 & $\mathbf{2 3}$ \\
\hline
\end{tabular}

\begin{tabular}{|c|c|c|c|c|c|}
\hline $\mathbf{2}$ & 6 & 1 & 2 & 3 & 1 \\
\hline 3 & $\mathbf{1 6}$ & 5 & 1 & 5 & 4 \\
\hline 1 & 0 & $\mathbf{4}$ & 0 & 1 & 0 \\
\hline 0 & 1 & 1 & $\mathbf{1}$ & 0 & 0 \\
\hline 0 & 4 & 0 & 0 & $\mathbf{1}$ & 1 \\
\hline 0 & 0 & 0 & 0 & 0 & $\mathbf{2 3}$ \\
\hline
\end{tabular}

CoR

\begin{tabular}{|c|c|c|c|c|c|}
\hline $\mathbf{6}$ & 4 & 0 & 1 & 4 & 0 \\
\hline 10 & $\mathbf{9}$ & 0 & 5 & 10 & 0 \\
\hline 0 & 0 & $\mathbf{6}$ & 0 & 0 & 0 \\
\hline 1 & 0 & 0 & $\mathbf{0}$ & 2 & 0 \\
\hline 2 & 1 & 0 & 0 & $\mathbf{3}$ & 0 \\
\hline 0 & 0 & 0 & 0 & 0 & $\mathbf{2 3}$ \\
\hline
\end{tabular}

SC-AL

\begin{tabular}{|c|c|c|c|c|c|}
\hline $\mathbf{3}$ & 3 & 0 & 0 & 9 & 0 \\
\hline 5 & $\mathbf{1 0}$ & 2 & 2 & 15 & 0 \\
\hline 0 & 0 & $\mathbf{6}$ & 0 & 0 & 0 \\
\hline 1 & 0 & 0 & $\mathbf{0}$ & 2 & 0 \\
\hline 1 & 0 & 0 & 0 & $\mathbf{5}$ & 0 \\
\hline 0 & 0 & 0 & 0 & 0 & $\mathbf{2 3}$ \\
\hline
\end{tabular}

$\mathrm{CD}$

\begin{tabular}{|c|c|c|c|c|c|}
\hline $\mathbf{6}$ & 2 & 3 & 1 & 3 & 0 \\
\hline 6 & $\mathbf{1 0}$ & 5 & 3 & 10 & 0 \\
\hline 0 & 2 & $\mathbf{4}$ & 0 & 0 & 0 \\
\hline 1 & 0 & 1 & $\mathbf{1}$ & 0 & 0 \\
\hline 0 & 0 & 2 & 0 & $\mathbf{3}$ & 1 \\
\hline 0 & 0 & 0 & 0 & 0 & $\mathbf{2 3}$ \\
\hline
\end{tabular}

qualities. The columns of the confusion matrices represent the predicted clusters while the rows represent the intended classes. From the diagonal entries of these matrices (which are the numbers of objects that have been correctly identified for each class), it is clear that SC-SR best reveals the six classes in the ground truth data.

\section{RELATED WORK}

In this section we give a review of the literature that is related to our work. We start with techniques in the general field of graph-based data processing and learning problems. Next, we move onto spectral methods applied on graphs. Finally, we discuss several existing works that involve the framework of combining information from multiple graphs.

Nowadays, graph theory is widely considered as a powerful mathematical tool for data modeling and processing, especially when the pairwise relationships between objects are of interest. Over the last two decades, graph-based data mining and analysis have become extremely popular. In Cook et al. [32] the authors have described the recent developments on the theoretical and practical aspects of graph-based data mining problems together with some practical applications. Especially, clustering on graphs has attracted a large amount of interests due to its numerous applications. In Schaeffer [1] the author has investigated the state-of-the-art techniques and recent advances in this active field, from hierarchical clustering to graph cuts, spectral methods and Markov chain based methods. These are certainly the foundations of our work. From a methodology point of view, regularization theory on graphs is of particular interest. In Smola et al. [33], the authors have developed the regularization theory for learning on graphs using the canonical family of kernels on graphs. In Zhou et al. [18], the authors have defined a family of differential operators on graphs, and used them to study the "smoothness" measure of functions defined on graphs. They have then proposed a regularization framework based on the developed smoothness measure. These works provide the main inspirations that lead to our second approach.

In addition to general graph-based data processing, we are especially interested in a unique branch in graph theory that is devoted to analyzing the spectrum of the graphs, namely, spectral graph theory. The manuscript of Chung [14] gives a good introduction to this field. Among various methods that have been developed, we particularly emphasize the socalled spectral clustering algorithm, which has become one of the major graph-based clustering techniques. Due to its promising performance and close links to other well-studied mathematical problems, a large number of variants of the original algorithm has been proposed, such as the constrained spectral clustering algorithms [34]-[38]. In general, these works have suggested different ways to incorporate constraints in the clustering task. Among them, Li et al. [36] has proposed a regularization framework in the graph spectral domain, which provides the closest methodology to our work.

Recently, data that can be represented by multiple graphs has attracted increasing attention. In the literature of the learning community this is sometimes referred to as "multiple views" or "multiple kernels", which intuitively means we investigate data from different viewpoints. In this setting, the general problem is how to efficiently combine information from multiple graphs for the learning objectives. In this sense, the following research efforts have the closest ideas to our presented work. In Argyriou et al. [39], the authors have proposed a method to compute an "optimal combined kernel" for combining graphs. Their idea is essentially based on averaging the graph Laplacian matrices. In Zhou et al. [40], the authors have modeled spectral clustering on a single graph as a random walk process, and then proposed a mixed random walk when two graphs are given. However, the way they make the combination is still based on a convex combination of the two graphs. In Muthukrishnan et al. [41], the authors have presented a novel way to exploit the relationships between different graph layers, which permits efficient combination of multiple graphs by a regularization framework in the signal domain. In Cheng et al. [42], the authors have proposed to achieve the final clustering result by post-processing the results from individual graph layers. In Savas et al. [43] and Vasuki et al. [44], the authors have worked with very similar settings to our work, however the problems they have tackled there are not clustering. Finally, the work of Tang et al. [13] is the closest to our first algorithm SC-GED in the sense that they have also proposed a unified matrix factorization framework 
to find a joint low dimensional representation shared by the multiple graphs, which inspired us to develop our first approach. Very recently, Kumar et al. [5] has proposed the co-regularization framework which is conceptually similar to our second algorithm SC-SR, and is adopted as a competing method in our experiments.

To summarize, although some of the works mentioned above are closely related to what we have presented in this paper, there are still noticeable differences that can be summarized as follows. First, despite the nature of the spectral clustering algorithm, most of the existing efforts to combine information from multiple graph layers are done in the signal domain, while the well-developed spectral techniques are mostly applied on a single graph. In contrast, our proposed methods provide novel ways for the same task in the graph spectral domain. Second, to the best of our knowledge, in almost all the state-of-the-art algorithms for combining multiple graphs, different graph layers are either treated equally or combined through a weighted summation. However, we propose SC-SR based on a spectral regularization process, in which individual graph layers play different roles in the combination process. In addition, we suggest to quantitatively measure the respective importance of different graph layers from an information-theoretic point of view, which could be beneficial for processing multiple graphs in general. Third, there are only a few works that address the problem of clustering with multiple graph layers, especially in the context of mobile social network analysis. We believe that our efforts to work with rich mobile phone datasets are good attempts in this emerging field.

\section{CONCLUSION}

In this paper we study the problem of clustering with data that can be represented by multi-layer graphs. We have shown that generalizations of the well-developed spectral techniques applied on a single graph are of great potential in such emerging tasks. In particular, we have proposed two novel methodologies to find a joint spectrum that is shared by all the graph layers: a joint matrix factorization approach and a graph-based spectral regularization framework. In the second approach, we suggest to treat individual graph layers based on their respective importance, which is measured from an information-theoretic point of view. In addition to the improvements we get in the evaluation scores with three social network datasets, we believe that the concept of a joint spectrum shared by multiple graphs is of broad interest in graph-based data processing tasks, as it suggests one way to generalize the classical spectral analysis to multi-dimensional cases. This is certainly one of the focuses in our future work.

\section{ACKNOWLEDGEMENT}

This work has been partly funded by Nokia Research Center (NRC), Lausanne, Switzerland. The authors would like to thank Prof. Radu Horaud for pointing out references [43], [44], and Prof. Nathan Eagle for the permission of using his figure in this paper (Fig. 1). The authors are also grateful to Prof. Weinman for the kind sharing of the MATLAB implementation of the L-BFGS algorithm ${ }^{7}$.

\footnotetext{
${ }^{7}$ http://www.cs.grinnell.edu/ weinman/code/index.shtml
}

\section{REFERENCES}

[1] E. Schaeffer, "Graph clustering," Computer Science Review, vol. 1, no. 1, pp. 27-64, 2007.

[2] N. Eagle, A. Clauset, A. Pentland, and D. Lazer, "Multi-Dimensional Edge Inference," Proceedings of the National Academy of Sciences (PNAS), vol. 107, no. 9, p. E31, 2010.

[3] N. Eagle and A. Pentland, "Reality Mining: Sensing Complex Social Systems," Personal and Ubiquitous Computing, vol. 10, no. 4, pp. 255268,2006

[4] J. Shi and J. Malik, "Normalized Cuts and Image Segmentation," IEEE Trans. Pattern Anal. and Mach. Intell., vol. 22, no. 8, pp. 888-905, Aug 2000.

[5] A. Kumar, P. Rai, and H. Daumé III, "Co-regularized Spectral Clustering with Multiple Kernels," NIPS 2010 Workshop: New Directions in Multiple Kernel Learning, 2010.

[6] N. Eagle, A. Pentland, and D. Lazer, "Inferring Social Network Structure Using Mobile Phone Data," in Proceedings of the National Academy of Sciences, vol. 106, no. 36, 2009, pp. 15 274-15278.

[7] U. von Luxburg, "A Tutorial on Spectral Clustering," Statistics and Computing, vol. 17, no. 4, pp. 395-416, 122007.

[8] J. B. MacQueen, "Some Methods for classification and Analysis of Multivariate Observations," Proceedings of 5th Berkeley Symposium on Mathematical Statistics and Probability, p. pp. 281297, 2010.

[9] L. Lovász, "Random Walks on Graphs: A Survey," Combinatorics, Paul Erdös is Eighty, vol. 2, pp. 353-398, János Bolyai Mathematical Society, Budapest, 1996.

[10] G. W. Stewart and J. Sun, "Matrix Perturbation Theory," Academic Press, New York, 1990.

[11] R. Bhatia, "Matrix Analysis," Springer, New York, 1997.

[12] J. Nocedal and S. J. Wright, "Numerical Optimization," Springer Series in Operations Research and Financial Engineering, 2006.

[13] W. Tang, Z. Lu, and I. Dhillon, "Clustering with Multiple Graphs," in International Conference on Data Mining, Miami, Florida, USA, Dec 2009.

[14] F. Chung, "Spectral Graph Theory," American Mathematical Society, 1997.

[15] R. P. Horaud, "A Short Tutorial on Graph Laplacians, Laplacian Embedding, and Spectral Clustering," http://csustan.csustan.edu/ tom/ Lecture-Notes/Clustering/GraphLaplacian-tutorial.pdf.

[16] D. Knossow, A. Sharma, D. Mateus, and R. P. Horaud, "Inexact matching of large and sparse graphs using laplacian eigenvectors," in Proceedings 7th Workshop on Graph-based Representations in Pattern Recognition, ser. LNCS 5534. Venice, Italy: Springer, May 2009. [Online]. Available: http://perception.inrialpes.fr/Publications/ 2009/KSMH09

[17] A. Sharma, R. P. Horaud, D. Knossow, and E. von Lavante, "Mesh segmentation using laplacian eigenvectors and gaussian mixtures," in Proceedings of AAAI Fall Symposium on Manifold Learning and its Applications, ser. Fall Symposium Series Technical Reports. Arlington, VA: AAAI Press, November 2009. [Online]. Available: http://perception.inrialpes.fr/Publications/2009/SHKV09a

[18] D. Zhou and B. Schölkopf, "A Regularization Framework for Learning from Graph Data," in ICML Workshop on Statistical Relational Learning and Its Connections to Other Fields, 2004, pp. 132-137.

[19] D. Zhou, O. Bousquet, T. N. Lal, J. Weston, and B. Schölkopf, "Learning with Local and Global Consistency," in Advances in Neural Information Processing Systems (NIPS), vol. 16, Cambridge, MA, 2004, pp. 321328. (Eds.) S. Thrun, L. Saul and B. Schölkopf, MIT Press.

[20] X. Zhu, Z. Ghahramani, and J. Lafferty, "Semi-supervised learning using Gaussian fields and harmonic functions," in The 20th International Conference on Machine Learning (ICML), 2003.

[21] O. Bousquet, O. Chapelle, and M. Hein, "Semi-supervised learning using Gaussian fields and harmonic functions," in Advances in Neural Information Processing Systems (NIPS), vol. 16, Cambridge, MA, 2004, pp. (Eds.) S. Thrun, L. Saul and B. Schölkopf, MIT Press.

[22] D. Zhou, J. Weston, A. Gretton, O. Bousquet, and B. Schölkopf, "Ranking on Data Manifolds," in Advances in Neural Information Processing Systems (NIPS), vol. 16, Cambridge, MA, 2004, pp. 169176. (Eds.) S. Thrun, L. Saul and B. Schölkopf, MIT Press.

[23] V. R. de Sa, "Spectral Clustering with two views," in Proceedings of the Workshop on Learning with Multiple Views, ICML, 2005.

[24] N. Kiukkonen, J. Blom, O. Dousse, D. Gatica-Perez, and J. Laurila, "Towards Rich Mobile Phone Datasets: Lausanne Data Collection Campaign," in International Conference on Pervasive Services, Berlin, Germany, Jul 2010. 
[25] M. Girvan and M. E. J. Newman, "Community Structure in Social and Biological Networks," Proceedings of the National Academy of Sciences (PNAS), vol. 99, pp. 7821-7826, 2002.

[26] M. E. J. Newman and M. Girvan, "Finding and evaluating community structure in networks," Phys. Rev. E, vol. 69, p. 026113, 2004.

[27] M. E. J. Newman, "Fast algorithm for detecting community structure in networks," Phys. Rev. E, vol. 69, p. 066133, 2004.

[28] N. Nefedov, "Multi-Membership Communities Detection in Mobile Networks," in Workshop of the International Conference on Web Intelligence, Mining and Semantics, May 2011.

[29] V. D. Blondel, J.-L. Guillaume, R. Lambiotte, and E. Lefebvre, "Fast unfolding of communites in large networks," Journal of Statistical Mechanics: Theory and Experiment, vol. 1742-5468, no. P10008, p. (12 pp.), 2008.

[30] C. D. Manning, P. Raghavan, and H. Schütze, "Introduction to Information Retrieval," Cambridge University Press, 2008.

[31] R. B. Lehoucq and D. C. Sorensen, "Deflation Techniques for an Implicitly Re-Started Arnoldi Iteration," SIAM J. Matrix Analysis and Applications, vol. 17, pp. 789-821, 1996.

[32] D. Cook and L. Holder, "Mining Graph Data," Wiley, December 2006

[33] A. Smola and R. Kondor, "Kernels and Regularization on Graphs," in 16th Annual Conference on Computational Learning Theory, Washington, DC, USA, Aug 2003.

[34] Q. Xu, M. desJardins, and K. Wagstaff, "Constrained Spectral Clustering under a Local Proximity Structure," in Proceedings of the 18th International Florida Artificial Intelligence Research Society (FLAIRS) Conference, May 2005.

[35] Z. Lu and M. Á. Carreira-Perpiñán, "Constrained spectral clustering through affinity propagation," in IEEE Conf. Computer Vision and Pattern Recognition, 2008.

[36] Z. Li, J. Liu, and X. Tang, "Constrained clustering via spectral regularization," in IEEE Conf. Computer Vision and Pattern Recognition, 2009.

[37] Z. Li and J. Liu, "Constrained Clustering by Spectral Kernel Learning," in Proc. IEEE Int'l Conf. Computer Vision (ICCV), 2009.

[38] X. Wang and I. Davidson, "Flexible constrained spectral clustering," in ACM SIGKDD Conference on Knowledge Discovery and Data Mining, Washington, DC, Jul 2010, pp. 563-572.

[39] A. Argyriou, M. Herbster, and M. Pontil, "Combining Graph Laplacians for Semi-Supervised Learning," in NIPS, 2005.

[40] D. Zhou and C. Burges, "Spectral Clustering and Transductive Learning with Multiple Views," in Proceedings of the 24th International Conference on Machine Learning (ICML), 2007, pp. 1159-1166.

[41] P. Muthukrishnan, D. Radev, and Q. Mei, "Edge Weight Regularization Over Multiple Graphs For Similarity Learning," in IEEE International Conference on Data Mining, Sydney, Dec 2010.

[42] Y. Cheng and R. Zhao, "Multiview spectral clustering via ensemble," in IEEE International Conference on Granular Computing, Nanchang, Aug 2009, pp. 101-106.

[43] B. Savas, W. Tang, Z. Lu, and I. S. Dhillon, "Supervised Link Prediction Using Multiple Sources," in IEEE International Conference on Data Mining, Sydney, Dec 2010.

[44] V. Vasuki, N. Natarajan, Z. Lu, and I. S. Dhillon, "Affiliation Recommendation using Auxiliary Networks," in Proceedings of the 4th ACM Conference on Recommender Systems(RecSys), Sep 2010.

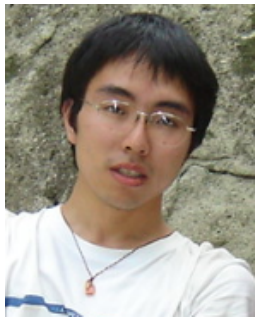

Xiaowen Dong has been working as a PhD student in the Signal Processing Laboratories (LTS4 / LTS2) at Swiss Federal Institute of Technology (EPFL), Lausanne, Switzerland since October 2009. His research interests include wavelets, spectral graph theory, and their applications to mobile and online social network analysis

Before joining EPFL, Mr. Dong received his B.Eng. degree in Information Engineering from Zhejiang University, Hangzhou, China and his M.Sc. degree in Signal Processing from Institute for Digital Communications, The University of Edinburgh, Edinburgh, UK.

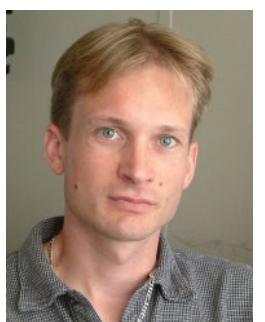

Pascal Frossard (S96,M01,SM04) received the M.S. and Ph.D. degrees, both in electrical engineering, from the Swiss Federal Institute of Technology (EPFL), Lausanne, Switzerland, in 1997 and 2000, respectively. Between 2001 and 2003, he was a member of the research staff at the IBM T. J. Watson Research Center, Yorktown Heights, NY, where he worked on media coding and streaming technologies. Since 2003, he has been a faculty at EPFL, where he heads the Signal Processing Laboratory (LTS4). His research interests include image representation and coding, visual information analysis, distributed image processing and communications, and media streaming systems.

Dr. Frossard has been the General Chair of IEEE ICME 2002 and Packet Video 2007. He has been the Technical Program Chair of EUSIPCO 2008 , and a member of the organizing or technical program committees of numerous conferences. He has been an Associate Editor of the IEEE TRANSACTIONS ON MULTIMEDIA (2004-), the IEEE TRANSACTIONS ON IMAGE PROCESSING (2010-) and the IEEE TRANSACTIONS ON CIRCUITS AND SYSTEMS FOR VIDEO TECHNOLOGY (2006-2011). He is the Vice-Chair of the IEEE Image, Video and Multidimensional Signal Processing Technical Committee (2007-). He is an elected member of the IEEE Visual Signal Processing and Communications Technical Committee (2006-) and of the IEEE Multimedia Systems and Applications Technical Committee (2005-). He has served as Vice-Chair of the IEEE Multimedia Communications Technical Committee (2004-2006) and as a member of the IEEE Multimedia Signal Processing Technical Committee (2004-2007). He received the Swiss NSF Professorship Award in 2003, the IBM Faculty Award in 2005, the IBM Exploratory Stream Analytics Innovation Award in 2008 and the IEEE Transactions on Multimedia Best Paper Award in 2011.

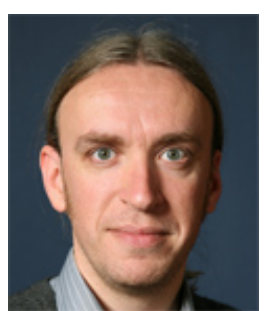

Pierre Vandergheynst received the M.S. degree in physics and the Ph.D. degree in mathematical physics from the Université catholique de Louvain, Louvain-la-Neuve, Belgium, in 1995 and 1998, respectively. From 1998 to 2001, he was a Postdoctoral Researcher and an Assistant Professor with the Signal Processing Laboratory, Swiss Federal Institute of Technology (EPFL), Lausanne, Switzerland. He is now an Associate Professor at EPFL, where his research focuses on harmonic analysis, sparse approximations, and mathematical image processing with applications to higher dimensional, complex data processing.

Dr. Vandergheynst was co-Editor-in-Chief of Signal Processing from 2002 to 2006 and has been an Associate Editor of the IEEE TRANSACTIONS ON SIGNAL PROCESSING since 2007. He has been on the Technical Committee of various conferences and was Co-General Chairman of the EUSIPCO 2008 conference. He is the author or co-author of more than 50 journal papers, one monograph and several book chapters. He is a laureate of the Apple ARTS award and holds seven patents.

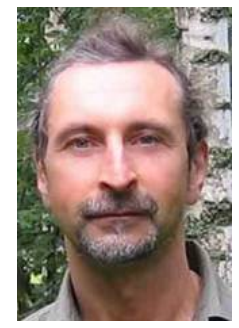

Nikolai Nefedov received M.Sc. degree in RadioPhysics with Honors (1982) from St. Petersburg State Technical University and Ph.D. degree in Communications Theory (1989) from St. Petersburg State University of Telecommunications (Russia). During 1982-1985 and 1988-1993 he worked at different research positions in Russian Academy of Science in Institute of Nuclear Physics (St. Petersburg) and Institute of Atmospheric Physics (Moscow). He was with ATMEL Development Center/Finland during 1993-1997, where he was involved in ASIC and DSP design. During 1996-2005 he hold different positions in Helsinki University of Technology, Adjunct Professor since 2004. He joined Nokia Research Center in 1997, where he is currently Principal Scientist. Since 2005 he is also affiliated with ISI Laboratory, ETH Zurich.

Dr. Nefedov has been on the Technical Committee of various conferences in field of wireless communications and networks, he is the author or co-author of more than 50 publications and 16 patents and patent applications. His current activities include interdisciplinary research in field of statistical physics, largescale dynamical systems, machine learning, and distributed algorithms. 